\title{
Barriers and Challenges to Telecardiology Adoption in Malaysia Context
}

\author{
Kee-Jiar Yeo ${ }^{1}$, Lina Handayani ${ }^{2}$, Haikal Satria ${ }^{3}$, Shih-Hui Lee ${ }^{4}$, Chia-Keat Yap ${ }^{5}$ \\ 1,4,5 Faculty of Education, Universiti Teknologi Malaysia, Johor, Malaysia \\ ${ }^{2}$ Faculty of Public Health, Universitas Ahmad Dahlan, Yogyakarta, Indonesia \\ ${ }^{3}$ Faculty of Biosciences \& Medical Engineering, Universiti Teknologi Malaysia, Johor, Malaysia
}

\begin{tabular}{l} 
Article Info \\
\hline Article history: \\
Received Dec 29, 2016 \\
Revised Jan 15, 2016 \\
Accepted Jan 30, 2016 \\
\hline Keyword: \\
Cardiac care \\
Cardiovascular \\
Telecardiology \\
Telecardiology adoption \\
Telemedicine
\end{tabular}

\begin{abstract}
Mainly in infrastructure deficient communities, telecardiology is considered as a complement to insufficient cardiac care. Telecardiology can reduce travelling and waiting time, enables information sharing in shorter time and facilitate care in rural and remote areas. A qualitative study examined the perspectives of health care providers: cardiologist and general physician and health care service receivers: patient and public towards telecardiology adoption. The barriers in telecardiology adoption were identified in this paper. It includes practicality of telecardiology, the need of education for staffs and administrators, ease of use, preferred face-to-face consultation, cost and confidentiality. Improvements can be done by the implementers based on this study in order to promote telecardiology successfully in Malaysia.

Copyright $(2017$ Institute of Advanced Engineering and Science. All rights reserved.
\end{abstract}

\section{Corresponding Author:}

Kee Jiar Yeo,

Faculty of Education,

Universiti Teknologi Malaysia,

Johor, Malaysia.

Email: kjyeo.utm.my

\section{INTRODUCTION}

Cardiovascular disease was ranked as the top cause of mortality according to the report from World Health Organization that last updated on May 2014 [1]. It caused 29,363 or $23.10 \%$ of total deaths and leading other diseases such as stroke, influenza, lung disease, road traffic accidents and so on in the causation of mortality in Malaysia.

Malaysia had been facing the scarcity of cardiologists and specialists for a long period. The overall density of cardiologists to the population was 0.07 per 10,000 populations, that were only 199 cardiologists in whole Malaysia. The number was greatly lower than the recommendations of Royal College of Physicians and British Cardiac Society, which is one cardiologist per 50,000 populations. According to the data, only 49 cardiologists out of the 199 cardiologists are working in public hospitals. The rest are serving in Ministry of Defense, universities, and private hospitals. Moreover, most of the cardiologists are located in urban area has caused the rural areas facing greater challenges in approaching cardiologists [2].

As early as 1997, the Ministry of Health $(\mathrm{MoH})$ in Malaysia had decided to develop telehealth and issued its official documents "Telemedicine Blueprint: Leading Healthcare into Informational Age" [3]. Malaysia is one of the pioneers among the developing countries which decided to adopt telehealth as a solutions for the problems that mushrooming in the healthcare industries. Telemedicine project was launched in Malaysia as one of the national flagship applications in April 2000 with vision to expand the clinical service virtually and expertise to rural and remote areas thus to improve the quality of national health care. Telecardiology is a mechanism that delivers cardiovascular services by using information and 
communication technology (ICT) at distance. It serves as a modern medical practice that provides remote diagnosis and treatment of heart disease with the use of telecommunications.

Previous studies had identified three groups of individuals: direct telemedicine providers, primary care practitioners and administrators are those who can affect the degree and the rate of telecardiology adoption [4]. However, the perspectives of patients and publics in telecardiology are not examined. Therefore, in this qualitative study, the perspectives of health care providers: cardiologist and general physician and health care service receiver: patient and public towards adoption of telecardiology were examined.

\section{LITERATURE REVIEW}

Telemedicine refers to the application of technologies for the transfer of clinical information. Telemedicine covered four subcomponents; include teleconsultation, health online, continuing professional development and lifetime health record service. The introduction of the internet has enabled telemedicine to expand its service to other medical specialty. Its use in cardiology is termed "telecardiology". Telecardiology is a mechanism that delivers cardiovascular services by using information and communication technology (ICT) at distance. Previous studies also argued that telecardiology system in Malaysia is essential to overcome the shortage of cardiology service in rural area. The system also will assist cardiologist to remote monitoring their patients in rural area and remote supervising the medical doctors at public non-specialist hospitals [5].

The main beneficiaries of the service are patients, healthcare professionals, the healthcare providers, health authority and the public. Telemedicine project was launched in Malaysia as one of the national flagship applications in April 2000 with vision to expand the clinical service virtually and expertise to rural and remote areas thus to improve the quality of national health care [2],[6]. The first telecardiology project was implemented in 38 Ministry of Health $(\mathrm{MOH})$ hospitals and health clinics across the country (mainly Peninsular Malaysia) over a 30-month period. However, telecardiology was not embraced nationwide due to some implementation and adoption barriers such as technological problem, insufficient ICT infrastructure and inconsistency of demand although the implementation had gone through evolution of massive technological and infrastructure alteration and upgrades as to uphold the providers in promoting good health and preventing mortality and morbidity.

A study by Molinari [7] had shown significant result on the use of telecardiology service can reduce the number of unnecessary hospital referrals and admissions. This enables minimizing travel, minimizing the length of hospital stays, reducing waiting list of patients, and preventing duplications in clinical work, examinations and test. Based on the study of Molinari et al. in 2002, out of 322 patients deemed not to have had an acute coronary event, 56 patients (17\%) were discovered to have ECG testing and a tele-consultation with a cardiologist. Thirty-eight of these patients were diagnosed subsequently with an acute coronary syndrome. Therefore, application of telecardiology can assist physicians to make accurate decision when facing with acute situation as the patient information are kept in computer and is accessible to the physicians or other health care providers. Telecardiology is also convenient to be used. It is more convenient for the patients to undergo ECG-testing in one's own home or local general health care centers [8]. From practitioners' perspectives, telecardiology can improve access to health care services for population living in remote areas.

However, implementation of telecardiology has its shortcomings related to cost, appropriateness, infrastructure and acceptance. Pak [9] argued that there is no significant difference in direct cost when teledermatology was compared with the normal casual visits. Neesa [10] indicated that patients in Bangladesh who can travel within two hours to main hospital prefer to physically meet the doctor. They prefer face-to-face consultation rather than to use the technology at the nearest health care center. Broens [11] had identified the determinants that would influence future implementation of telemedicine and the determinants are technology, acceptance, financing, organization and policy. Parasuraman [12], [13] argued that exploration of users' readiness is significant due to increasing incidence of customers having to serve themselves through technology-based systems is germane not only to service companies but also to goods companies.

The perceptions of patients and carers towards the services were studied in order to understand their satisfaction towards the services. Based on past research, the satisfaction level of users' towards healthcare services is significant in promoting cooperation with that care and increase compliance with treatment. Besides that, it also helps health professionals to identify problems in healthcare and evaluate the care, to understand the communication process between doctors and patients, predict patient health-related behavior and to predict patient utilization of care, continuity with the same provider and compliance [14]. 


\section{METHODS}

In this qualitative study, non-probability sampling was used to select the population for study. Samples were selected deliberately in order to answer the research questions. Semi-structured interviews were conducted with cardiologist, general physicians, patient and public so that their opinions towards telecardiology adoption can be understood. Semi-structured interviews often provide a reliable and comparable data. It also allows exploration of individual experiences whilst ensuring consistency across respondents. Interviewees' consent was obtained before the face-to-face interviews which took approximately 20-30 minutes.

The interviews were conducted between July 2016 and August 2016. A cardiologist from private sector, a general physician, a patient who suffered from cardiovascular disease and an adult from the public were interviewed. The interviewer informed the purpose of the study and explained the term 'telecardiology' in the context of this study to the interviewees prior to the interview. The interview questions focused on the advantages of using telecardiology whether in interviewees' workplace practice or self-check health routine and the barriers might existed for telecardiology adoption. The results were recorded, transcribed and thematically coded.

\section{RESULTS AND DISCUSSIONS}

The general rational of this study was to obtain opinions on telecardiolgy adoption among the public and the stakeholders of cardiovascular sector as shown in Table 1. The interviews were transcribed and themes that emerged from the data were identified.

Table 1. Opinions on Telecardiolgy Adoption

\begin{tabular}{lllll}
\hline & & \multicolumn{2}{c}{ Healthcare Providers } & \multicolumn{2}{c}{ Healthcare Receivers } \\
\hline Advantages & a. & Reduce travelling time and cost & a. & Reduce travelling and waiting time \\
& b. & Sharing of information in shorter period & b. & Facilitates care in rural and remote areas \\
Barriers & c. & Facilitates care in remote and rural area & & \\
& a. & Practicality of telecardiology & a. & Accessibility of the service \\
& b. & Education for staff and administrators is & b. & Education for staff and administrators is \\
& & needed & c. & Ease of use \\
& c. & Face-to-face consultation is preferred & d. & Preferred in-person consultation \\
& d. & Cost for adopting telecardiology & e. & Cost for adopting telecardiology \\
e. & Confidentiality & &
\end{tabular}

\subsection{The Perspectives of Healthcare Providers \\ 4.1.1. Reduce Travelling Time and Cost}

Cardiologist believed that by using telecardiology can reduce the travelling time for specialists to attend conference regarding the issues. He suggested that decentralized meeting and information sharing through video conferencing can be done in order to reduce the cost of travelling, accommodation and increase their efficiency at the same time. "The doctors can resume to work immediately after they attended the meeting in the morning instead of spending few days on travelling, attending the meeting and so on."

\subsubsection{Sharing of Information in Shorter Period}

Cardiologist and general physician also agreed that the information of the patient or clinical subject can be shared effectively. At the meanwhile, general physicians also mentioned that with the use of telecardiology, the communications among the staffs in the departments can be done in higher efficiency. Currently, communication application, for instance WhatsApp is served as a useful tool for communication among the cardiologists and general physicians. Pictures, recorded videos, electrocardiogram (ECG) and echocardiograms can be sent through the application that enables them to discuss and seek for professional opinions.

\subsubsection{Facilitates care in remote and rural area}

Cardiologist mentioned in the interview, telecardiology might be more useful in rural and remote area of Malaysia. "In the urban area, such as Johor Bahru and Kuala Lumpur, getting consultation and treatment from specialists can be done easily as most of the specialists are distributed in cities." The cardiologist revealed that the distribution of cardiologist throughout the country is not even, most of the cardiologist preferred to serve in urban area. Additional to that, the facilities and medical equipment in remote areas are insufficient. Therefore, telecardiology might be more useful in remote and rural areas. 


\subsubsection{Practicality of Telecardiology}

During the interview, the cardiologist had questioned the practicality of telecardiology in Malaysia setting. He mentioned in the interview, although telecardiology can forward the information of a patient to the control center and request for consultant, however the information could be inaccurate based on the patient's description and even ECG can be misleading sometimes.

Besides that, he also stressed on the point that face-to-face consultation is irreplaceable in diagnosis of cardiovascular diseases. The public interviewee also concerned about the design and how telecardiology works. "Is the service available 24 hours? Is there anyone to answer my inquiry even I call in midnight?" The availability of service throughout the day also one of the concern for cardiologist, patient and the public as the timing for patient inquiry is unpredictable and can be happened at any time.

Addition to that, general physician also pointed out that even though most of the areas in Malaysia are equipped with internet/ telco coverage, however, there are some hospitals in rural or remote areas still lack of internet coverage and even ICT technology that can support telecardiology services.

\subsubsection{Education for Staff and Administrators is Needed}

Healthcare providers stressed that the training for staff before telecardiology adoption is important because they are the front line of the service. The cardiologist said, "The training and education for the healthcare staffs is very significant, as some of the time ECG and the evidences given by the patients can be misleading and make inappropriate judgments."

\subsubsection{Face-to-face Consultation is preferred}

The cardiologist had mentioned face-to-face consultation still the best approach to diagnose cardiovascular disease among the patients as there are some symptoms cannot be detect by merely using ECG or echocardiograms. Therefore, professionalism of the cardiologist is irreplaceable by adopting telecardiology.

\subsubsection{Cost for Adopting Telecardiology}

General physician pointed out in the interview, the cost for ICT infrastructure and other additional expenses might be another barrier in telecardiology adoption. She mentioned that the infrastructure for some of the hospitals is not complete and inadequate to support the systems. Therefore, a big amount of money needs to be invested in order to develop the service.

\subsubsection{Confidentiality}

Confidentiality of the patient information was one of the minor themes identified from the interviews. The cardiologist has shown a lot of concern towards the confidentiality of the telecardiology system as the data can be access in a more open manner that might arise ethical issues.

\subsection{The Perspectives of Healthcare Receivers}

\subsubsection{Reduce Travelling and waiting Time}

Patient and public also indicated that waiting time can be reduced if telecardiology can be used to identify emergency cases and receive treatment as soon as possible. As one of the patient revealed that the waiting time for receiving treatment in general hospital was quite long based on his own experience. Tests were conducted repetitively in order to verify the condition of the patients. Before the patient sent to the general hospital, he had went to the private hospital to do his checkup, the doctor-in-charge had referred the patient to the general hospital which equipped with cardiology service at that time. Although he was referred to emergency department, however, the patient had to wait for quite a long time before admission. "I spent one whole day to wait for the admission and around 10 days until I received the surgery," said the patient. Therefore, he hoped the waiting time and referral process can be greatly reduced and simplified if telecardiology is adopted.

\subsubsection{Facilitates Care in Rural and Remote Areas}

This common theme was identified from both healthcare providers and receivers. The public interviewee had pointed out that seeking a specialist in Johor Bahru area is very convenient. Therefore, telecardiology is more likely to be useful in remote and rural area.

\subsubsection{Accessibility of the Service}

The public interviewee concerned about the design and how telecardiology works. "Is the service available 24 hours? Is there anyone to answer my inquiry even I call in midnight?" The availability of 
service throughout the day also one of the concern for patient and the public as the timing for patient inquiry is unpredictable and can be happened at any time.

\subsubsection{Education for staff and Administrators is needed}

The healthcare receivers had shown concern about the quality of the staff and administrators of telecardiology services. They worried about the handling process and how the staffs going to make appropriate judgment and suggestions based on the conditions described through the phones or other ICT devices.

\subsubsection{Ease of Use}

The public interviewee had mentioned the design of telecardiology devices need to be easy and convenient to use in order to cope with emergency case. Both patient and the public interviewee agreed that complicated operations could be a barrier for older people to adopt telecardiology in their healthcare routine. Besides that, based on their suggestions, the devices used to contact the control center need to be easy to bring along and if possible can send emergency call to the control center when the patient is at risk.

\subsubsection{Preferred in-Person Consultation}

The patient and the public interviewee also indicated that in-person consultation is more trustworthy and more comfortable. The patient noted that explaining the conditions through phone call might be difficult and can create confusion between both parties. At the meanwhile, they also agreed that meeting the cardiology specialist in person can give them more confidence towards the service provided.

\subsubsection{Cost for Adopting Telecardiology}

Patient and public interviewee also concerned about the charges of the services, as most medical services available in the market are not cheap. They had questioned about the availability of subsidy when applying telecardiology service. "What is the charges we need to pay if we are using the service? Is this subsidized by the government?" The public interviewee enquired.

Based on previous research, the adoption and rate of use of telecardiology or other telemedicine services are affected by many factors. In this study, we intended to shed some light on the perceptions of health care providers: cardiologist and general physician and the potential service receiver: patient and public towards the use of telecardiology. The interviewees generally responded positively towards telecardiology. The advantages identified from the interviews are reduces travelling time and waiting time, enables sharing of information in shorter period, and facilitates care in remote and rural area. Our findings supported the idea mentioned by Backman [8] that telecardiology will help in empowering the general physicians as it provides diagnostic support that enabling them to safely manage more of their own patients.

However, they also had some hesitations towards the adoption of telecardiology. Their perception is related to the attitude of acceptance as pointed out by Davis [15] that high perceived usefulness and perceived ease of use will contribute to higher level of technology acceptance. The perceived barriers were summarized by respondents includes practicality of telecardiology, the need for education for staff, ease of use, preferred face-to-face consultation, cost and confidentiality. Broens [11] had identified the determinants that would influence future implementation of telemedicine and the determinants are technology, acceptance, financing, organization and policy. Besides that, the results was supported by Hoop [4]. The barriers to telemedicine implementation included technical challenges, the need for more education and training for patients and staff, preferences for in-person care, the need for program improvement and the need for additional time to provide telemedicine services [4].

As mentioned in Collins and his colleagues' study [14], the future success of telemedicine services will depend on patient and providers acceptance of such technologies. The findings from the study suggest the aspects that can be revised and improved by the telecardiology services providers and implementers. For instance, the practicality of the service, to ensure the service can be provided continually, a complete system of operations need to be work out. The availability of ICT infrastructure or the bandwidth of internet need to be upgraded, so that images and information sent to the control center or cardiologists could be used for reliable diagnosis. Ajzen [16] also indicated the more resources and opportunities individuals believe they have, and the fewer obstacles or impediments they anticipate, the greater should be their perceived control over the behavior.

Besides that, education program focused on operations of telecardiology for the staff and implementers are essential. The quality of the healthcare providers has an effect on people's confidence towards the service. At the same time, telecardiology can be used as a tool for continuous professional development of the staff from time to time. Discussions and training can be conducted by using videoconferencing to support staff learning. General physicians in Scotland have used telemedicine as an access to 
educational resources. The physicians in remote areas had seen telemedicine as opportunities to access educational events, as it reduced the cost of travelling and also increase the accessibility in remote areas [17].

Last but not least, the education for the publics can be significant in successful adoption of telecardiology. Although telecardiology program had been launched in Malaysia for quite some times, but it is still very new to the public as most of the interviewees mentioned that they never heard telecardiology before. Therefore, the education of telecardiology for the public could increase the understanding of public towards the technology and thus aids in expansion of telecardiology in Malaysia.

\section{CONCLUSION}

This study reveals that there is still a limit to the advantages of telecardiology. The participants have reservation on its appropriateness and ease of use even though they acknowledged the conveniences and other benefits in telecardiology. With entire dependent on technology, telecardiology will not be the preferred choice and patients will be undiagnosed or even untreated if there is no access to the internet or patients are not ready to accept such technology. Nevertheless, this study has its limitation in sample size. Future research should include more subjects and may include quantitative data collection through survey method. Besides that, since telecardiology program had not been fully implemented in Malaysia, the perception of the healthcare providers and receivers could be not precise towards the use of telecardiology. Further examinations from varying context need to carry out in order to provide empirical evidence.

\section{ACKNOWLEDGEMENTS}

This work was supported by grant from the Ministry of Higher Education (MOHE), Trans Disciplinary Research Grant Scheme (TRGS): PY/2016/06027 cost centre: R.J130000.7831.4L848.

\section{REFERENCES}

[1] H. Wang, et al., "Global, regional, and national life expectancy, all-cause mortality, and cause-specific mortality for 249 causes of death, 1980-2015: a systematic analysis for the Global Burden of Disease Study 2015," The Lancet, vol. 388, pp. 1459-1544, 2015.

[2] N. Maarop, et al., "Exploring factors that affect teleconsultation adoption: In the case of Malaysia," 2011.

[3] D. P. Sugijarto, et al., "A Case Study on Laboratory Information System,” Procedia Technology, vol. 11, pp. 740$747,2013$.

[4] F. Hopp, et al., "Perspectives from the Veterans Health Administration about opportunities and barriers in telemedicine," Journal of telemedicine and telecare, vol. 12, pp. 404-409, 2006.

[5] H. Yew, et al., "A Telecardiology Framework for Rural Area," WSEAS, pp. 140-148, 2014.

[6] N. Maarop and K. T. Win, "Understanding the Need of health care providers for teleconsultation and technological attributes in relation to the acceptance of teleconsultation in Malaysia: A mixed methods study," Journal of medical systems, vol. 36, pp. 2881-2892, 2012.

[7] G. Molinari, et al., "The role of telecardiology in supporting the decision-making process of general practitioners during the management of patients with suspected cardiac events," Journal of telemedicine and telecare, vol. 8, pp. 97-101, 2002.

[8] W. Backman, et al., "The telecardiology revolution: improving the management of cardiac disease in primary care," Journal of the Royal Society of Medicine, vol. 103, pp. 442-446, 2010.

[9] H. S. Pak, et al., "Cost minimization analysis of a store-and-forward teledermatology consult system," Telemedicine and e-Health, vol. 15, pp. 160-165, 2009.

[10] A. Nessa, et al., "Applicability of telemedicine in Bangladesh: current status and future prospects," in Convergence and Hybrid Information Technology, 2008. ICCIT'08. Third International Conference on, pp. 948-953, 2008.

[11] T. H. Broens, et al., "Determinants of successful telemedicine implementations: a literature study," Journal of telemedicine and telecare, vol. 13, pp. 303-309, 2007.

[12] A. Parasuraman, "Technology Readiness Index (TRI) a multiple-item scale to measure readiness to embrace new technologies," Journal of service research, vol. 2, pp. 307-320, 2000.

[13] A. Parasuraman and C. L. Colby, "Techno-ready Marketing: How and Why Your Customers Adopt Technology," New York, Free Press, 2001.

[14] K. Collins, et al., "Patient satisfaction in telemedicine," Health Informatics Journal, vol. 6, pp. 81-85, 2000.

[15] F. D. Davis, "Perceived usefulness, perceived ease of use, and user acceptance of information technology," MIS quarterly, pp. 319-340, 1989.

[16] I. Ajzen, "The theory of planned behavior," Organizational behavior and human decision processes, vol. 50, pp. 179-211, 1991.

[17] G. King, et al., "Adoption of telemedicine in Scottish remote and rural general practices: a qualitative study," Journal of telemedicine and telecare, vol. 13, pp. 382-386, 2007. 